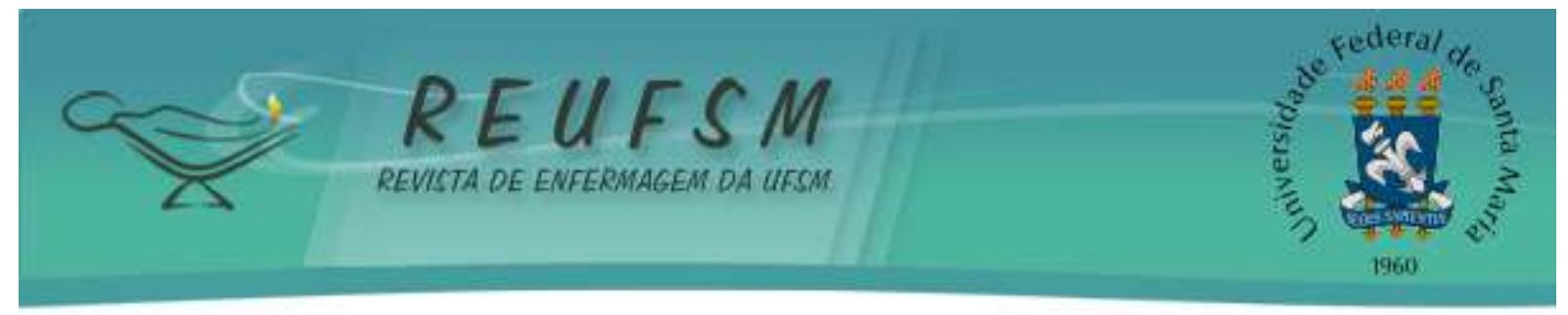

\title{
PRÁTICAS DOS ENFERMEIROS ÀS PESSOAS EM CRISE PSÍQUICA EM DECORRÊNCIA DO USO ABUSIVO DE DROGAS
}

\section{PRACTICES OF NURSES TOWARDS PEOPLE EXPERIENCING PSYCHIC CRISIS RESULTING FROM DRUG ABUSE}

\section{PRÁCTICAS DE LOS ENFERMEROS A LAS PERSONAS EN CRISIS PSÍQUICA A CAUSA DEL USO ABUSIVO DE DROGAS}

Doi: 10.5902/2179769229596

\author{
Deivson Wendell da Costa Lima ${ }^{1}$ \\ Mirya Karol Brasil Serafim ${ }^{2}$ \\ Francisco Rafael Ribeiro Soares ${ }^{3}$ \\ Antônia Líria Feitosa Nogueira Alvino ${ }^{4}$ \\ Alcivan Nunes Vieira ${ }^{5}$ \\ Magda Fabiana do Amaral Pereira ${ }^{6}$
}

RESUMO: Objetivo: compreender as práticas dos enfermeiros em serviço de urgência e emergência às pessoas em situação de crise psíquica decorrente do uso abusivo de drogas. Método: pesquisa descritiva, abordagem qualitativa realizada com 19 enfermeiros que trabalham no pronto-socorro de um hospital geral no interior do Rio Grande do Norte. Os dados foram coletados através de uma entrevista semiestruturada, e após foram submetidos à análise de conteúdo. Resultados: os enfermeiros enfatizam que suas práticas são focadas nos sinais e sintomas da crise psíquica ou usam a escuta como instrumento de prática. Além disso, relataram que as dificuldades enfrentadas nas práticas são o preconceito ao usuário de drogas, a falta de capacitação na área de saúde mental e a estrutura física inadequada do hospital. Conclusão: evidenciou-se a necessidade de qualificação do enfermeiro para cuidar de pessoas que fazem uso abusivo de drogas e maior envolvimento dos serviços que compõem a Rede de Atenção Psicossocial.

Descritores: Enfermagem; Prática profissional; Transtornos relacionados ao uso de substâncias; Emergência.

\footnotetext{
1 Enfermeiro. Doutorando pelo Programa de Pós-Graduação em Enfermagem Psiquiátrica da Escola de Enfermagem de Ribeirão Preto da Universidade de São Paulo. Docente da Faculdade de Enfermagem da Universidade do Estado do Rio Grande do Norte. Mossoró, RN, Brasil. E-mail: deivsonwendell@ @otmail.com

${ }^{2}$ Enfermeira. Graduada em Enfermagem pela Universidade do Estado do Rio Grande do Norte. Mossoró, RN, Brasil. E-mail: karolbrasil.11@gmail.com

${ }^{3}$ Enfermeiro. Doutorando pelo Programa de Pós-graduação Cuidados Clínicos em Enfermagem e Saúde da Universidade Estadual do Ceará. Docente da Faculdade de Enfermagem da Universidade do Estado do Rio Grande do Norte. Mossoró, RN, Brasil. E-mail: soaresfrr@gmail.com

${ }^{4}$ Enfermeira. Doutoranda pelo Programa de Pós-graduação Psicobiologia da Universidade Federal do Rio Grande do Norte. Docente da Faculdade de Enfermagem da Universidade do Estado do Rio Grande do Norte. Mossoró, RN, Brasil. E-mail: antonialiria@gmail.com

5 Enfermeiro. Doutor pelo Programa de Pós-graduação Cuidados Clínicos em Enfermagem e Saúde da Universidade Estadual do Ceará. Docente da Faculdade de Enfermagem da Universidade do Estado do Rio Grande do Norte. Mossoró, RN, Brasil. E-mail: alcivan_nunes@yahoo.com.br

${ }^{6}$ Enfermeira. Mestre pelo Programa de Pós-graduação em Saúde Pública da Universidade Federal do Ceará. Docente da Faculdade de Enfermagem da Universidade do Estado do Rio Grande do Norte. Mossoró, RN, Brasil. E-mail: magdafabiana@gmail.com
} 


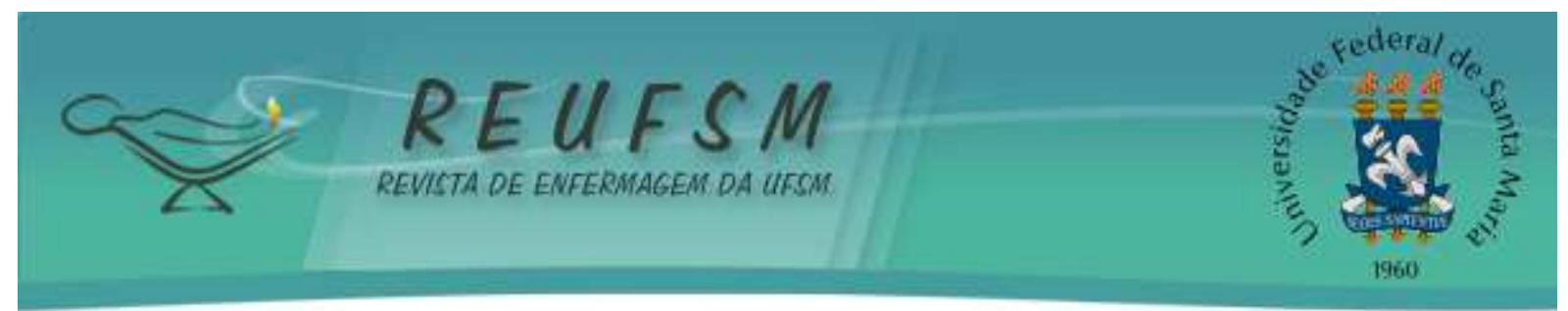

ABSTRACT: Aim: to understand the practices of nurses in urgent and emergency service for people experiencing psychic crisis resulting from drug abuse. Method: this is a descriptive research, with a qualitative approach, performed with 19 nurses who work in the emergency room of a general hospital in the countryside of Rio Grande do Norte. Data were collected through semi-structured interviews, and then submitted to content analysis. Results: nurses highlight that they focus on the signs and symptoms of the psychic crisis or use listening as an instrument of practice, and they also reported that the difficulties faced by them are the the prejudice against the drug user, the lack of training in the field of mental health and the inappropriate physical structure of the hospital. Conclusion: there is a need for nurses' qualification so that they can care for people who abuse drugs and for a greater engagement of the services that comprise the Psychosocial Care Network.

Descriptors: Nursing; Professional Practice; Substance-Related Disorders; Emergencies.

RESUMEN: Objetivo: comprender las prácticas utilizada por los enfermeros, en un servicio de urgencia y emergencia, a las personas en situación de crisis psíquica a causa del uso abusivo de drogas. Método: investigación descriptiva, de base cualitativa, realizada con 19 enfermeros que trabajan en el espacio de primeros auxilios de un hospital general en el interior de Rio Grande do Norte. Los datos fueron recolectaron por medio de entrevista semiestructurada y después sometidos a análisis de contenido. Resultados: los enfermeros señalan que sus prácticas se centran en los signos y síntomas de la crisis psíquica o usan la escucha como instrumento de práctica, además relatan que las dificultades enfrentadas en las prácticas son el prejuicio contra el usuario de drogas, la falta de capacitación en el área de salud mental y la estructura física inapropiada del hospital. Conclusión: se evidenció la necesidad de calificación del enfermero para cuidar de las personas que hacen uso abusivo de drogas y mayor comprometimiento de los servicios que componen la Red de Atención Psicosocial.

Descriptores: Enfermería; Práctica Profesional; Trastornos Relacionados con Sustancias; Urgencias Médicas.

\section{INTRODUÇÃO}

A Reforma Psiquiátrica Brasileira constituiu-se em um movimento social, jurídico e político que contribuiu para a desconstrução da proposta de internação manicomial como estratégia central para o tratamento das pessoas com transtornos mentais. Este movimento foi reafirmado com a Lei 10.206/01 que prevê a construção de novas alternativas de atenção à saúde mental baseadas na lógica da cidadania. Em consequência disso, houve a ampliação dos serviços comunitários oferecidos e maiores investimentos na área de saúde mental. ${ }^{1}$

Após 10 anos, instituiu-se no âmbito do Sistema Único de Saúde (SUS) a portaria $\mathrm{N}^{\mathrm{o}}$ 3.088, a qual estabelece a Rede de Atenção Psicossocial (RAPS) para atendimento das pessoas com transtornos mentais e/ou com necessidades decorrentes do uso e abuso de drogas desde atenção básica até os hospitais gerais, de modo articulado e integrado com base no território. ${ }^{2}$ 


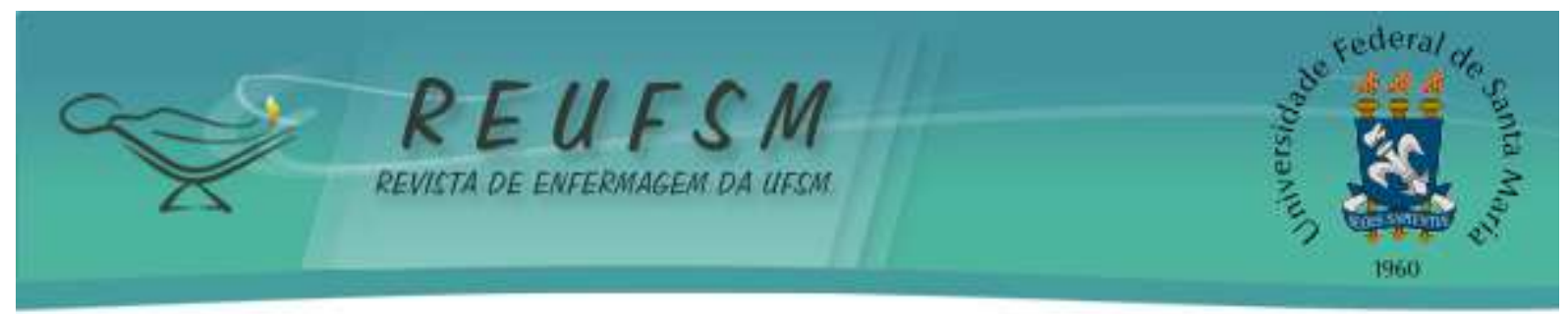

Em consonância com a realidade nacional, a implantação da RAPS no local deste estudo não aconteceu de forma plena e estruturada. A diminuição de 200 para 160 leitos no hospital municipal psiquiátrico e o fechamento do ambulatório, denominado Unidade Integrada de Saúde Mental, representaram mudanças significativas na reorientação da assistência municipal em saúde mental, tendo em vista que esses dispositivos não são reconhecidos como integrantes da RAPS. ${ }^{2}$

Ainda no cenário municipal, outros avanços no percurso da Reforma Psiquiátrica Brasileira são perceptíveis, como a existência de dois Centros de Atenção Psicossocial II (CAPS II), um Centro de Atenção Psicossocial infantil (CAPSi), um Centro de Atenção Psicossocial álcool e outras drogas III (CAPSad III), uma equipe de Consultório na Rua, atendimentos realizados pelo Serviço de Atendimento Médico de Urgência (SAMU), uma sala de estabilização em uma Unidade de Pronto de Atendimento, quatro leitos de saúde mental em um hospital geral, Unidades Básicas de Saúde e Núcleos de Apoio à Saúde da Família. Estes serviços requerem ações intersetoriais com outros dispositivos estratégicos da assistência social, educação e segurança pública, no sentido de garantir uma atenção integral às pessoas com transtorno mental que fazem uso abusivo de drogas. ${ }^{3}$ Considera-se uso abusivo de drogas o padrão de uso que causa danos agudos à saúde do usuário, intoxicações, síndrome de abstinência e comorbidades relacionadas à cronicidade do uso. ${ }^{4}$

Vale destacar que cerca de 250 milhões de pessoas no mundo, com idade entre 15 e 64 anos usaram, pelo menos, um tipo de droga em 2014. Desta população, aproximadamente 29 milhões de pessoas fazem consumo abusivo de drogas. ${ }^{5}$ Todavia é o álcool, droga lícita, considerada a principal responsável pela procura de tratamento. ${ }^{4}$

Estas informações demonstram a necessidade de uma RAPS organizada, estruturada e de profissionais qualificados para atenderem de forma segura, adequada e resolutiva aqueles que procuram os serviços de saúde, até mesmo nas situações de crise psíquica, por uso abusivo de drogas, em especial nos hospitais de urgência e emergência. ${ }^{6-7}$ Diante desse contexto, questiona-se: como são as práticas realizadas pelos enfermeiros às pessoas em situação de crise psíquica pelo uso abusivo de drogas em um serviço de urgência e emergência?

A assistência de enfermagem às pessoas que fazem uso abusivo de drogas é frequente no setor de urgência e emergência de hospitais gerais, um dos serviços de saúde constituinte da RAPS. Esse espaço é favorável para identificação de pessoas que fazem uso abusivo de 


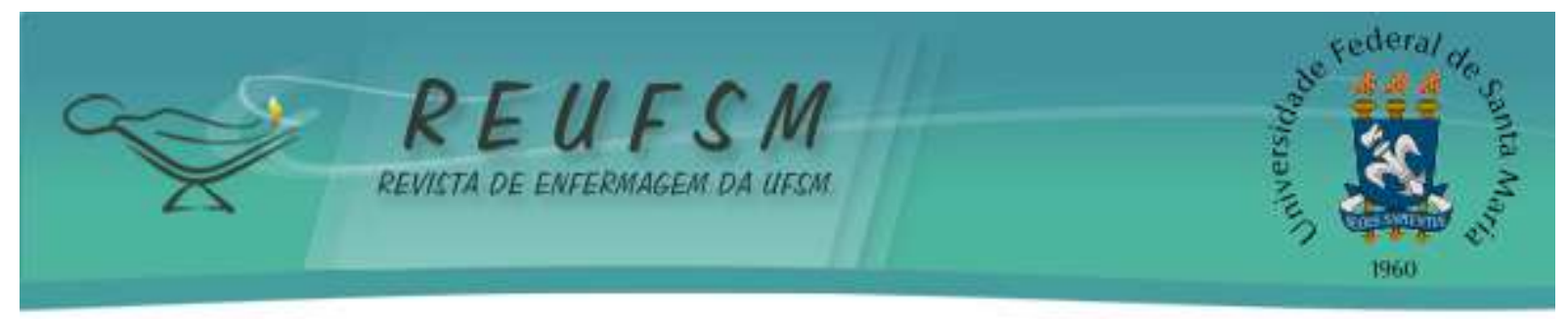

drogas ao possibilitar que os enfermeiros realizem intervenções imediatas e adequadas à situação da crise psíquica, minimizando os riscos e favorecendo o manejo clínico. ${ }^{8}$

Para tanto, os enfermeiros precisam ultrapassar os procedimentos técnicos e estabelecer intervenções promotoras da saúde mental, sem julgamentos e preconceitos, considerando e compreendendo que os significados atribuídos à droga relacionam-se, de maneira diferente, com a história de vida de cada indivíduo.

Nesta perspectiva, este estudo teve como objetivo compreender as práticas dos enfermeiros em serviço de urgência e emergência às pessoas em situação de crise psíquica decorrente do uso abusivo de drogas.

\section{MÉTODO}

Pesquisa descritiva de abordagem qualitativa desenvolvida em um hospital geral no interior do Rio Grande do Norte. É um hospital classificado como grande porte para atendimento de urgência e emergência do município sede e outras cidades circunvizinhas. A população desta pesquisa foram os enfermeiros que trabalham no pronto-socorro deste hospital, levando em consideração a amostragem por acessibilidade.

Quanto aos critérios de inclusão, ficou definido que o profissional deveria exercer a função de enfermeiro no pronto-socorro e trabalhar neste setor com período acima de três meses. E os critérios de exclusão foram ser enfermeiro apenas em cargos administrativos ou que estejam afastados do serviço por motivo de doença, férias, transferência ou greve. Dos 25 enfermeiros atuantes no pronto-socorro, 19 participaram da pesquisa, uma vez que quatro não aceitaram participar e dois estavam de licença do serviço.

Para a coleta de dados do estudo foi utilizado como técnica a entrevista semiestruturada que tinha como questão orientadora: fale-me sobre suas experiências no pronto-socorro com as pessoas que fazem uso abusivo de drogas em crise psíquica.

Os dados foram coletados no período de abril a julho de 2015, de acordo com a disponibilidade e concessão dos enfermeiros através do Termo de Consentimento Livre e Esclarecido (TCLE), após autorização da direção da instituição hospitalar e do responsável pelo setor.

Previamente foi realizada uma visita ao setor para entrega de uma carta convite aos enfermeiros do pronto-socorro da instituição. Neste dia, os enfermeiros foram informados 


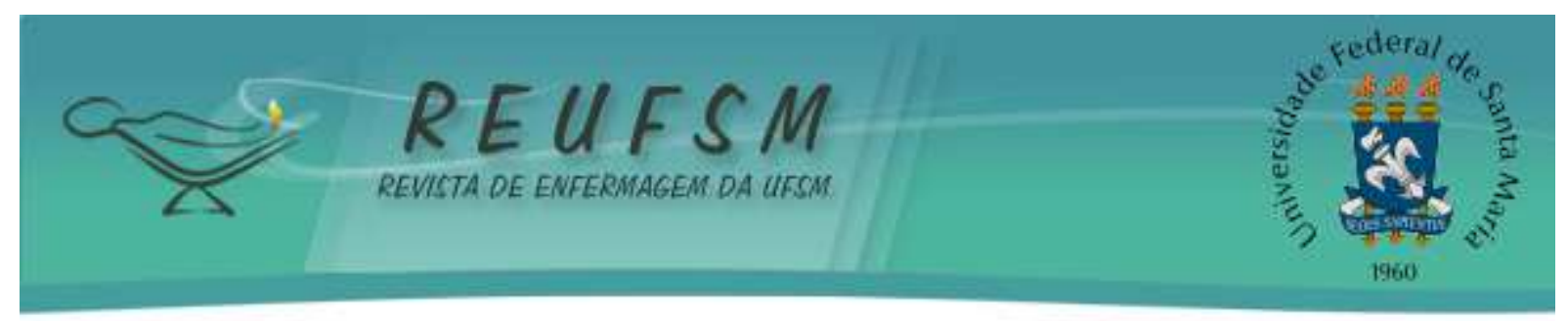

sobre o objetivo, a justificativa e a metodologia da pesquisa, além disso, foi explicado ao entrevistado que na execução do trabalho seria respeitado o anonimato. Aqueles que aceitaram participar da pesquisa foram agendados data, hora e local para a coleta dos dados, com objetivo de não interferir na dinâmica do seu trabalho.

As entrevistas foram realizadas em uma sala do hospital, local calmo e sem interrupções, mantendo a privacidade dos enfermeiros. As falas foram gravadas com duração média de 28 minutos e, posteriormente, transcritas na íntegra. Com o intuito de manter a preservação da identidade dos entrevistados e organização dos depoimentos, foi utilizada a letra "E" de enfermeiro, seguido de algarismo arábico para identificá-los.

O material empírico foi processado por meio de análise de conteúdo de Bardin, que se iniciou com a pré-análise, perpassando pela fase de exploração do material e finalizando com tratamento/interpretação dos resultados obtidos. Na primeira fase, foi realizada uma leitura geral das falas dos entrevistados, a fim de promover uma apropriação do conteúdo. Já a segunda fase consistiu em uma leitura mais aprofundada do material a ser analisado, que possibilitou a definição das categorias, em torno das quais as falas foram organizadas. $\mathrm{Na}$ terceira e última etapa, foram realizadas as interpretações do material analisado e estabelecidas às inferências, relacionando-as com os autores que auxiliam na discussão do estudo. ${ }^{9}$

A pesquisa seguiu os preceitos éticos da resolução no 466/12 do Conselho Nacional

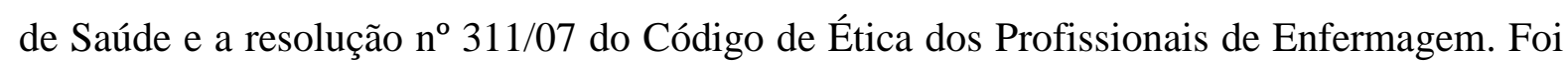
submetida à apreciação do Comitê de Ética em Pesquisa do Centro de Ensino Superior e Desenvolvimento - CESED, que emitiu parecer favorável em 02/02/2015, segundo protocolo CEP/CAAE - 39117114.2.0000.5294.

\section{RESULTADOS E DISCUSSÃO}

Os participantes foram 19 enfermeiros de ambos os sexos, 14 do sexo feminino e cinco do sexo masculino, com predominância da faixa etária de 31 a 40 anos, idade média de 36 anos. Eles trabalhavam no pronto-socorro do hospital com período de seis meses a 10 anos, média de tempo de serviço de dois anos e oito meses. Quanto à formação, 13 possuíam especialização, dois mestrado, um doutorado e três com graduação, sendo destes apenas dois enfermeiros tinham alguma formação na área de saúde mental. 


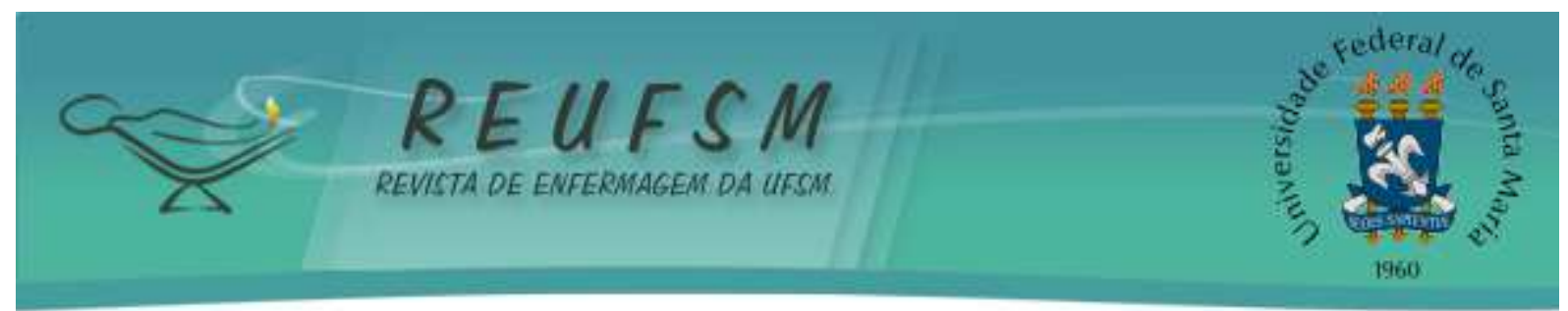

As falas dos entrevistados foram evidenciadas e organizadas em unidades de registro, de acordo com suas semelhanças e pertinência, que emergiu um quantitativo de 93 unidades de análise de conteúdo agrupadas em oito temas, que serão discutidos em duas categorias: práticas realizadas pelo enfermeiro à pessoa que faz uso abusivo de drogas; dificuldades nas práticas de enfermagem à pessoa que faz uso abusivo de drogas.

\section{Práticas realizadas pelo enfermeiro à pessoa que faz uso abusivo de drogas}

De um modo geral, as ações de enfermagem desenvolvidas no pronto-socorro junto a usuários de drogas em situação de crise psíquica são os atendimentos de classificação de risco, administração de medicações prescritas pelos médicos e a manutenção do quadro clínico conforme o agravo que o levou a procurar o serviço do hospital. Também foi evidenciado a escuta enquanto instrumento da prática de enfermagem, mas ainda é pouco desenvolvida por esses profissionais, seja por medo ou por não saberem lidar com um paciente em crise psíquica devido ao uso abusivo de drogas.

Identificou-se que a prática de enfermagem às pessoas em crise psíquica decorrentes do uso abusivo de drogas está pautada em uma lógica gerencial, controladora e prescritiva. Ocorre uma influência do modelo biomédico que repercute na mecanização do cuidado e fragmentação da pessoa em corpo e mente, normal e anormal. ${ }^{10}$

Percebeu-se a atuação dos enfermeiros no pronto-socorro com foco apenas nas dimensões anatômicas e fisiológicas do paciente, orientada pelas prescrições médicas e desconexa dos aspectos psicossociais. ${ }^{11} \mathrm{O}$ recorte da fala do entrevistado retrata essa realidade no hospital:

as nossas práticas são prestadas de acordo com a necessidade do paciente, é dado pelo sintoma. Se tiver com tremor e agitado, será feita a medicação conforme a prescrição. Se tiver desidratado, será feito o procedimento recomendado pelo médico. (E1)

A prestação de cuidados ao usuário de drogas deve ser realizada de forma crítica pelo enfermeiro, indo além do físico e não se detendo apenas em uma prescrição medicamentosa. Torna-se necessário uma prática voltada para o ser humano, sem pré concepções pessoais e sociais, com atenção na relação do uso da droga com o seu risco iminente de vida. ${ }^{12}$

No entanto, os enfermeiros buscam a solução da crise psíquica intervindo com uso da contenção mecânica ou química, como se pode ver na fala a seguir: 


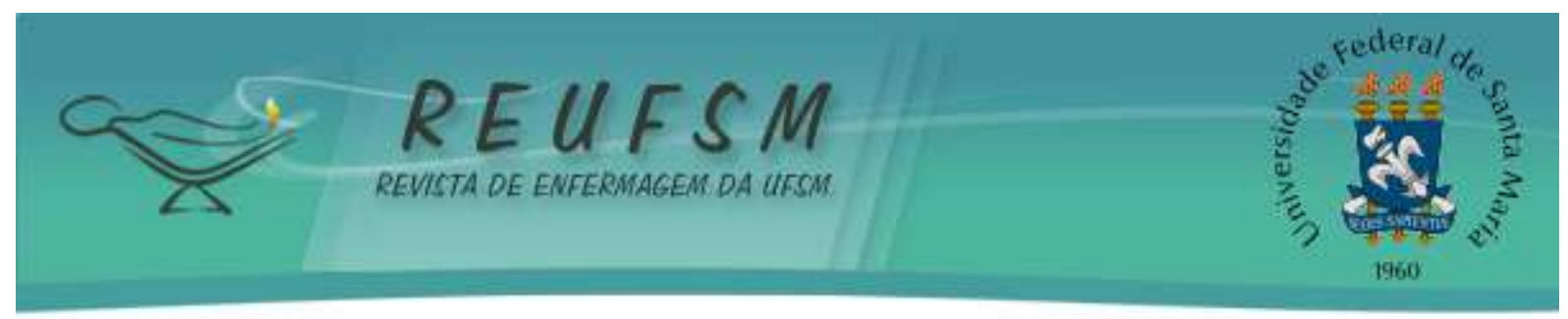

olhe, de modo geral, não existe nenhuma prática diferenciada para este tipo de paciente que chega aqui com alucinações $e$ incontrolável. Não tem nenhuma área específica para tratamento do usuário de drogas aqui. Se esse paciente está agitado, nós fazemos o procedimento, a contenção medicamentosa ou mecânica, contenção no leito. É o que temos aqui. (E6)

Fica evidente na fala do entrevistado que as contenções parecem ser a saída mais viável e fácil nas situações em que os pacientes se encontram agitados, em períodos de alucinações e delírios. Esses tipos de contenções são práticas tradicionais da enfermagem frequentes na área de urgência e emergência para lidar com comportamentos violentos e inadaptados, administrados em pacientes com transtornos mentais graves que devem ser mantidas apenas durante o período de risco. ${ }^{13-14} \mathrm{Em}$ países como a Noruega, a restrição física é rigorosamente regulada e monitorada para ser usada somente nas situações anteriormente mencionadas. ${ }^{15}$

Nessas ocasiões de crise psíquica devido o uso abusivo de drogas, é imprescindível a avaliação clínica rigorosa e pormenorizada da situação em que o paciente se encontra, analisando os riscos graves para ele próprio e/ou para outras pessoas envolvidas. ${ }^{11}$ Para tanto, faz-se necessário o uso gradativo de técnicas de comunicação pela enfermagem junto às pessoas que fazem uso de drogas, estimulando-os falar sobre seus sentimentos, sobre suas angústias, sobre seus significados e sua relação com a droga. ${ }^{16}$

A qualidade da abordagem inicial na emergência em saúde mental possui significativa influência sob a resposta do paciente ao trabalho da equipe, bem como na aceitação das recomendações e na sua adesão ao tratamento. Diante disso, se o cuidado em saúde mental ainda reproduz a atenção centrada na doença, isso quer dizer que os propósitos da Reforma ainda não foram alcançados. ${ }^{17}$

Os entrevistados também reconheciam que as práticas às pessoas em crise psíquica em decorrência do uso abusivo de drogas deveriam ser humanizadas, fazendo uso do instrumento terapêutico da escuta. Sobre isso relata o seguinte entrevistado:

estas pessoas querem ser consideradas como humanos, não apenas drogados. É preciso ter tolerância, paciência, usar técnicas melhores, escutar por que ele procurou ajuda aqui, se ele sabe as consequências da droga que usou. (E18)

A escuta é uma ferramenta da prática de enfermagem que possibilita desencadear respostas ou solucionar algum problema relatado pelas pessoas que fazem uso abusivo de drogas. Isso implica na necessidade na escuta das queixas, dos sofrimentos indicados pelo 


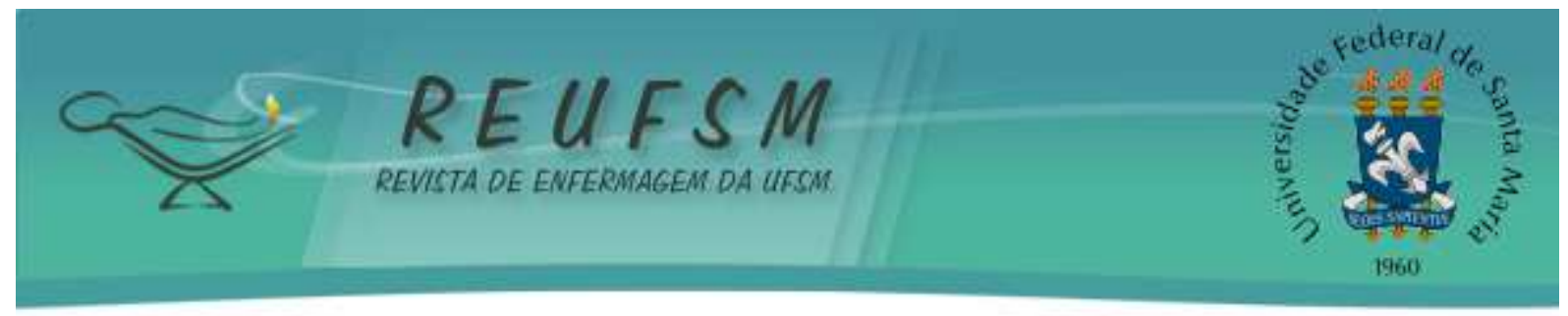

sujeito em busca de uma responsabilização pelas demandas pronunciadas. ${ }^{16}$ Nota-se a tentativa de realizar a escuta primeiro com a família do que com o paciente:

na minha prática, eu procuro escutar direto da família o que aconteceu com ele, depois procuro conversar com o paciente. (E2)

Os enfermeiros valorizam mais a queixa de quem trouxe a pessoa em crise psíquica para o serviço do que aquilo que essas pessoas têm a dizer de si mesmos. Mencionam a família como uma fonte privilegiada de informações e que é importante escutar estas pessoas. Contudo, o enfermeiro antes de escutar qualquer outra pessoa, precisa demonstrar atenção as queixas do próprio paciente, escutando o que ele tem a dizer. ${ }^{18}$

Também foi citado pelos entrevistados que a escuta da pessoa em crise psíquica devido ao uso abusivo de drogas, é responsabilidade dos profissionais do serviço social e da psicologia, como se pode perceber nas falas a seguir:

a gente conversa com eles e juntamente com o serviço social. Esses profissionais deveriam estar mais presentes aqui no pronto-socorro para escutar esta clientela. Se tem problema social, tem que ser resolvido com o assistente social. (E3)

chamo logo o psicólogo para tentar conversar, escutar, acalmar o paciente. Às vezes, escuto com ele para atender melhor o paciente. (E14)

Apesar da tentativa de alguns enfermeiros de realizar a escuta, a maioria dos entrevistados não compreendem a escuta como instrumento da prática de enfermagem em um serviço de urgência e emergência.

\section{Dificuldades nas práticas de enfermagem à pessoa que faz uso abusivo de drogas}

$\mathrm{Na}$ realidade do hospital geral deste estudo, os enfermeiros entrevistados mencionaram ter medo dos pacientes, associando a pessoa em crise psíquica devido ao uso abusivo de drogas ao estigma social de um suposto comportamento agressivo e assumem o preconceito diante da prestação do cuidado, como é percebido nas falas a seguir:

Eu tenho medo, mas a gente não nega a assistência, mas não vou ser hipócrita, a gente olha sim, com um certo preconceito. (E9)

Tem ainda um grande preconceito: Fulaninho é usuário de drogas. Aquele paciente ali, do leito tal, ele é usuário de drogas, então vamos ter cuidado, ele pode agredir alguém aqui. (E3)

Ainda existem muitas dificuldades de superar os valores culturais e os estigmas construídos no imaginário social relacionado às pessoas com transtornos mentais e que 


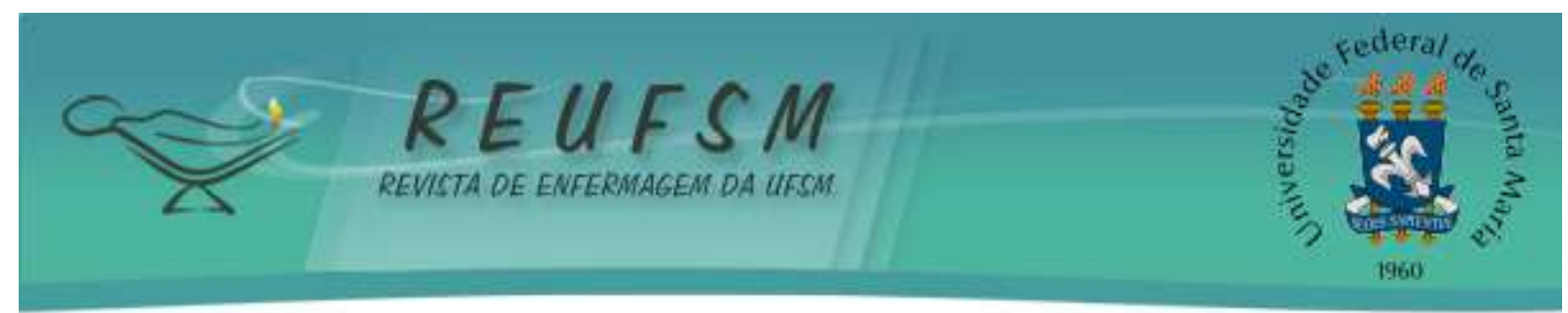

fazem uso e abuso de drogas, principalmente aqueles que fazem uso de drogas ilícitas. Isto reforça práticas excludentes que negligenciam as pessoas que fazem uso de drogas enquanto cidadãos, que possuem histórias de vida, desejos e necessidades de atenção e cuidado. ${ }^{19}$

Percebe-se que o preconceito relatado pelos enfermeiros dificulta a convivência dessas pessoas usuárias de drogas dentro de um mesmo pronto-socorro com pacientes que apresentam diferentes condições clínicas. Nesse sentindo, aquele em crise psíquica devido ao uso abusivo de drogas aparece nas falas como um problema, um transtorno, como uma situação complicada de imaginar a convivência entre esses pacientes.

Na minha concepção, você não pode pegar um paciente que está em surto ou em abstinência de droga e colocar ele com outros pacientes, com câncer, com infarto agudo do miocárdio. É um problema, é um transtorno. (E13)

Fica explícito no depoimento que as pessoas que fazem uso abusivo de drogas não deveriam ocupar o mesmo setor dos outros pacientes de patologias gerais. Nesse contexto, outro depoimento afirma que a condição de trabalho torna-se difícil quando um paciente em crise psíquica se encontra junto dos outros, pois além de ser difícil lidar com a situação, ainda pode haver uma não aceitação dos outros pacientes, como expôs o entrevistado:

a condição de trabalho é meio difícil, você ainda vai ter a dificuldade de lidar com esse paciente no meio dos outros, dependendo de como ele esteja e se os outros vão aceitar ou não. Muitos pedem para tirar essas pessoas de perto. (E6)

Segundo a opinião dos enfermeiros, identificou-se que a pessoa em crise psíquica e que faz uso abusivo de drogas não é tão aceita no pronto-socorro pelos enfermeiros e também pelos demais usuários daquele setor. Diante da política de saúde mental embasada na Reforma Psiquiátrica Brasileira, essa prática é considerada antiquada, experiência de exclusão social e de isolamento. ${ }^{19}$

Todos os atores envolvidos neste cenário precisam compreender que o hospital é um espaço social e de cuidado para todos, que eles estão se relacionando com pessoas e não com doenças. São necessárias práticas que usem os espaços hospitalares de acordo com a dinâmica da atenção psicossocial, em uma lógica na qual estimule a interação social, a humanização do cuidado e a convivência como favorecedores do processo terapêutico. ${ }^{20}$ 


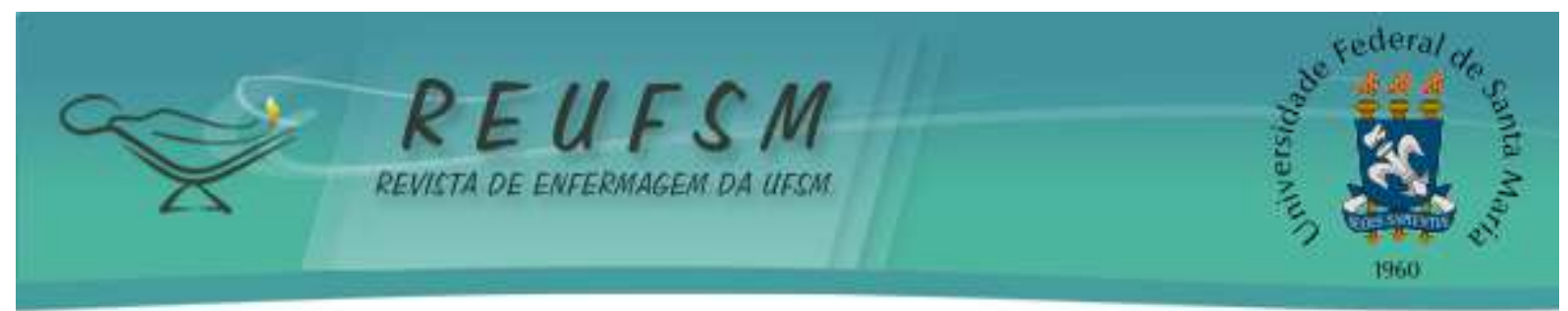

Outra dificuldade evidenciada nas falas dos enfermeiros foi à ausência de uma estrutura física adequada e a falta de insumos materiais que refletem nas suas condições de trabalho para atender a pessoa que faz uso abusivo de drogas:

hoje a condição de trabalho é zero, não temos estrutura física para trabalhar. Além disso, se você procurar no hospital um psicotrópico ou o mínimo do mínimo você não tem. O hospital ainda não está preparado para receber esses pacientes drogados em alucinação, em abstinência de crack. (E10)

O setor da saúde no Brasil ainda sofre por carência de recursos financeiros para suprir a falta de insumos, de profissionais bem remunerados, de leitos suficientes à demanda, de estrutura física adequada. Estes problemas dificultam a implementação e a execução efetiva das políticas públicas de saúde que repercutem no funcionamento dos hospitais gerais. ${ }^{21}$

Apesar desta situação, os enfermeiros mencionaram o desejo de uma nova realidade, com a existência de um setor específico no pronto-socorro, com leitos psiquiátricos, para atenção a esses pacientes em crise psíquica e o reconhecimento de uma atenção priorizada, como se pode vê na fala a seguir:

precisaria de toda uma estrutura e um atendimento especializado e voltado pra esse tipo de paciente aqui no pronto-socorro. A gente tem vontade, a gente queria uma ala específica para pacientes de saúde mental, seria bem diferente. (E5)

E é essa legislação vigente proposta pela Portaria ${ }^{\circ} .148$ de 2012 que regulamenta a organização do Serviço Hospitalar de Referência com estabelecimento de leitos integrais de saúde mental em hospitais gerais para atenção as pessoas com sofrimento mental e com necessidades decorrentes do uso e abuso de drogas. Essa Portaria orienta a criação de uma estrutura especializada para avaliação clínica e psicossocial por uma equipe multiprofissional, articulada com outros pontos de atenção da RAPS. ${ }^{22}$ Os números de leitos integrais de saúde mental não devem exceder 30 leitos do total de leitos do hospital. No entanto, têm-se apenas dois leitos psiquiátricos na clínica cirúrgica deste hospital. A falta de integração da rede de saúde é apontada pela maioria dos entrevistados como dificuldade para que ocorra a continuidade do cuidado a pessoa que faz uso abusivo de drogas.

Os enfermeiros demonstram desmotivação com o funcionamento da RAPS do lócus de atuação e do país como um todo. Afirmaram que não existe a continuidade do cuidado depois que o usuário deixa o hospital geral do munícipio, pois a rede de atenção à saúde encontra-se 


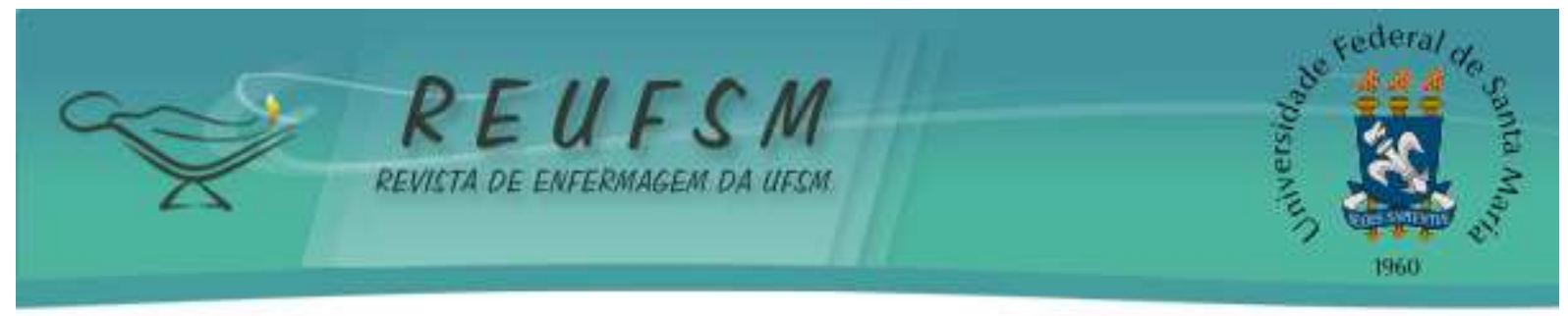

desarticulada, desorganizada, não há comunicação entre os serviços de saúde mental, de urgência e emergência e da atenção básica. Além disso, os profissionais desconhecem o funcionamento da RAPS, o que dificulta o compartilhamento de responsabilidades pelo cuidado da pessoa que faz uso abusivo de drogas.

É preciso estruturar a rede e a rede está bagunçada. Então, eu acho que a reforma falha muito nisso. (E4)

Não temos pra onde encaminhar ou como garantir um acompanhamento depois que ele sair daqui. (E12)

Torna-se necessário que os profissionais compreendam o conceito e o funcionamento da RAPS como o conjunto de ações e serviços de saúde articulados em níveis de complexidade crescente, na perspectiva de garantir a integralidade da assistência à saúde. A RAPS tem como objetivo oferecer um cuidado articulado, integrado e efetivo nos diferentes pontos de atenção da rede, atendendo as pessoas em sofrimento e com demandas decorrentes de transtornos mentais e de uso de drogas. ${ }^{2}$

Os enfermeiros criticam a aplicabilidade da Portaria 3.088 de 2011 quanto sua implantação da RAPS e percebem a necessidade de reestruturação do serviço para implantação de uma nova realidade que garanta um cuidado colaborativo e integral as pessoas que fazem uso abusivo de drogas.

A rede não está preparada. É preciso estruturar este serviço e os outros serviços, alinhar uns pontos para depois implementar um cuidado integral e potencial. (E2)

Os cuidados prestados aos usuários de drogas no hospital geral devem estar articulados com os demais serviços extra hospitalares existentes no território, tornando possível a promoção da corresponsabilidade, do acolhimento e da construção do vínculo entre o usuário e os profissionais do serviço de saúde. ${ }^{23}$

Os enfermeiros relataram que a falta de capacitação é considerada outra dificuldade para realizarem um cuidado de qualidade as pessoas em crise psíquica devido ao uso abusivo de drogas.

Eu acredito que se os profissionais tivessem um mínimo de capacitação teriam condições de oferecer um cuidado mais potencial. (E4)

Seria muito interessante ter cursos e treinamentos para a equipe, para mudar essa realidade. Se realmente acontecesse, o desafio maior seria colocar em pratica. (E8) 


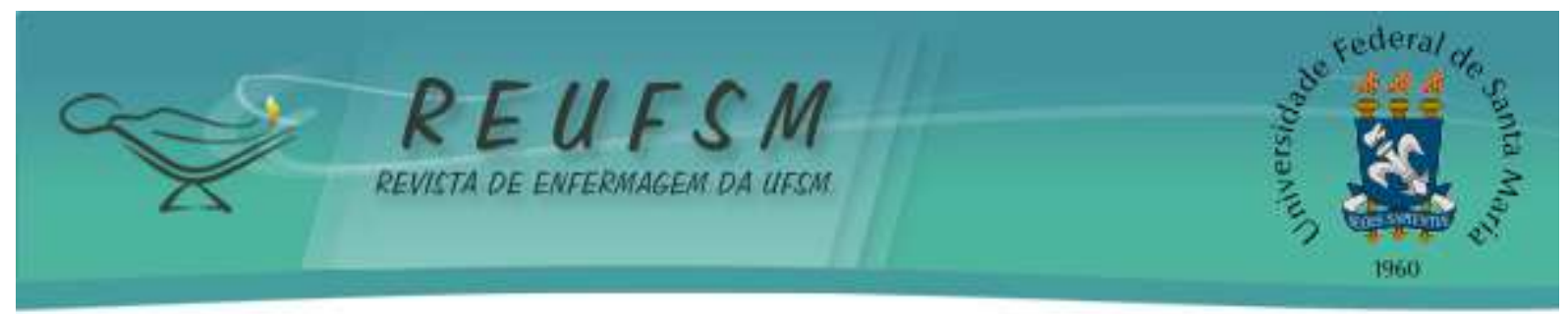

Os enfermeiros veem na capacitação uma forma de construir novas competências necessárias para atuarem junto às pessoas que fazem uso abusivo de drogas. No entanto, apenas dois enfermeiros entrevistados referiram formação em saúde mental. É fundamental provocar profissionais e gestores a reconhecerem no espaço de produção de cuidado, um ambiente de aprendizagem significativa, conectando-se ao ambiente de trabalho e conhecimentos nele presentes. ${ }^{24}$

No contexto da saúde, a capacitação no trabalho é considerada um instrumento que aponta alternativas para construção de novas competências e maior satisfação do profissional e do usuário. ${ }^{25}$ Compreende-se assim, que a capacitação torna-se um propulsor para a desconstrução do preconceito, instrumentaliza o enfermeiro para desenvolver práticas que considerem a história de vida das pessoas que fazem uso de drogas e que proporcionem a continuidade do seu tratamento na rede de atenção a saúde.

\section{CONSIDERAÇÕES FINAIS}

Essa pesquisa traz contribuição ao propor a discussão, pela voz dos enfermeiros de um hospital geral, que por trás da assistência prestada revelam seus medos, anseios e dificuldades. Aposta-se na necessidade de qualificação dos enfermeiros baseada na política nacional de saúde mental para que produzam, junto com outros profissionais, práticas efetivas colaborativas e interdisciplinar às pessoas em crise psíquica em decorrência do uso abusivo de drogas.

Além disso, considera-se pertinente enfatizar a necessidade de implantação de mais leitos integrais de saúde mental em hospitais gerais e maior envolvimento dos serviços que compõem a RAPS para garantia de um cuidado integral às pessoas que fazem uso abusivo de drogas Acredita-se também que ações de promoção à saúde mental podem sensibilizar os enfermeiros para o reconhecimento dos usuários de drogas como seres humanos e para o cuidado humanizado desenvolvido a eles.

Este estudo espera contribuir para o estimulo a novas e mais aprofundadas pesquisas na área de enfermagem no contexto da urgência e emergência, pois se verificou que as práticas tradicionais ainda não são suficientes para atender as necessidades daqueles usuários em uso abusivo, com possibilidade de apresentarem problemas mais graves relacionados às drogas. Daí a importância dos profissionais da saúde, especialmente o enfermeiro, investigarem de forma sistemática e sem julgamentos prévios a relação do usuário com as 


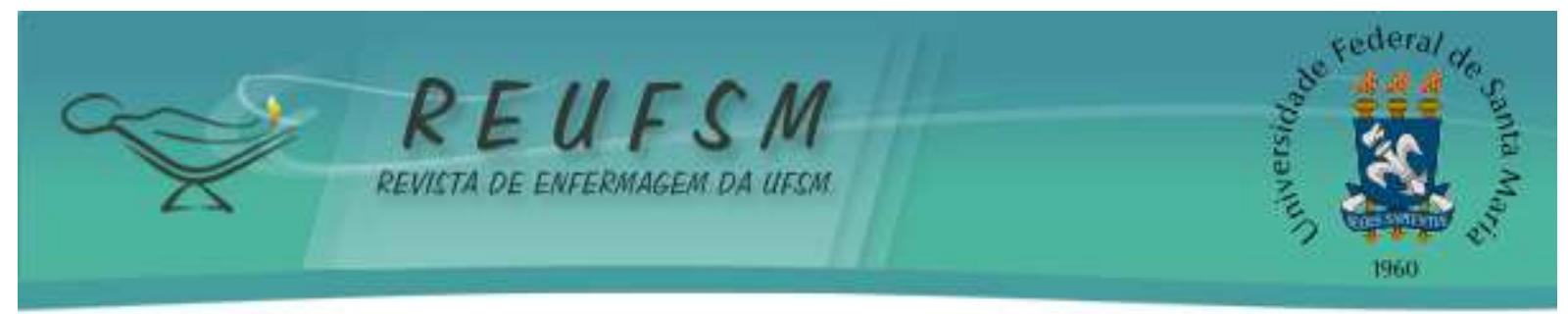

drogas, com isso prestarão uma assistência individualizada e de qualidade e contribuirão para diminuir na sociedade o preconceito em relação ao manejo do uso dessas substâncias.

Como limitação principal da pesquisa tem-se o fato da coleta ter sido realizada em um único serviço de urgência e emergência da RAPS e, nesse sentido, sugere-se que outros estudos possam ser realizados com foco nesta temática, tanto em nível local quanto nacional.

\section{REFERÊNCIAS}

1. Amarante P. Saúde mental e atenção psicossocial. $4^{\mathrm{a}}$ ed. Rio de Janeiro: Fiocruz; 2015. 123 p.

2. Brasil. Ministério da Saúde. Portaria n ${ }^{\circ}$ 3.088, de 23 de dezembro de 2011. Institui a Rede de Atenção Psicossocial para pessoas com sofrimento ou transtorno mental e com necessidades decorrentes do uso de crack, álcool e outras drogas, no âmbito do Sistema Único de Saúde (SUS). Brasília (DF): Ministério da Saúde; 2011[acesso em 2016 dez 10]. Disponível:http://bvsms.saude.gov.br/bvs/saudelegis/gm/2011/prt3088_23_12_2011_rep.html

3. Silva MNRMO, Santos V, Santos JE, Oliveira FM, Nogueira DJ, Galassi AD. Desenvolvendo e articulando a rede intersetorial para cuidado integral de usuários de drogas em contextos de vulnerabilidade. Cad Ter Ocup [Internet]. 2014 jul [acesso em 2016 dez 10];22(Supl Esp):145-52. Disponível em: http://www.cadernosdeterapiaocupacional.ufscar.br/index.php/cadernos/article/view/1045/524.

4. U.S. Departament of Health and Human Services. Substance Abuse and Mental Health Services Administration. Results from the 2013 National Survey on Drug Use and Health: summary of national findings [Internet]. NSDUH Series H-48, HHS Publication No. (SMA) 14-4863. Rockville, MD; 2014 [acesso em 2017 jan 20]. 184 p. Disponível em: https://www.samhsa.gov/data/sites/default/files/NSDUHresultsPDFWHTML2013/Web/NSD UHresults2013.pdf.

5. United Nations Office on Drugs and Crime (UNODC). World Drug Report 2012 [Internet]. United Nations publication; 2012 jun [acesso em $2016 \mathrm{dez}$ 15]. 101 p. Disponivel em: https://www.unodc.org/unodc/en/data-and-analysis/WDR-2012.html.

6. Almeida AB, Nascimento ERP, Rodrigues J, Schweitzer G. Intervenção nas situações de crise psíquica: dificuldades e sugestões de uma equipe de atenção pré-hospitalar. Rev Bras Enferm [Internet]. 2014 out [acesso em 2017 jan 20];67(5):708-14. Disponível em: http://www.scielo.br/pdf/reben/v67n5/0034-7167-reben-67-05-0708.pdf.

7. Zeferino MT, Cartana MHF, Fialho MB, Huber MZ, Bertoncello KCG. Percepção dos trabalhadores da saúde sobre o cuidado às crises na Rede de Atenção Psicossocial. Esc Anna Nery Rev Enferm [Internet]. 2016 [acesso em 2017 jan 20];20(3):e20160059. Disponível em: http://www.scielo.br/pdf/ean/v20n3/1414-8145-ean-20-03-20160059.pdf.

8. Prates JG, Pinho PH, Oliveira MAF, Claro HG. A concepção dos enfermeiros de serviços de urgência e emergência sobre o processo saúde-doença na assistência aos usuários de substâncias psicoativas. Saúde Debate [Internet]. 2014 jun [acesso em 2017 jan 20]; 38(101):318-27. Disponível em: http://www.scielo.br/pdf/sdeb/v38n101/0103-1104-sdeb-38-101-0318.pdf. 


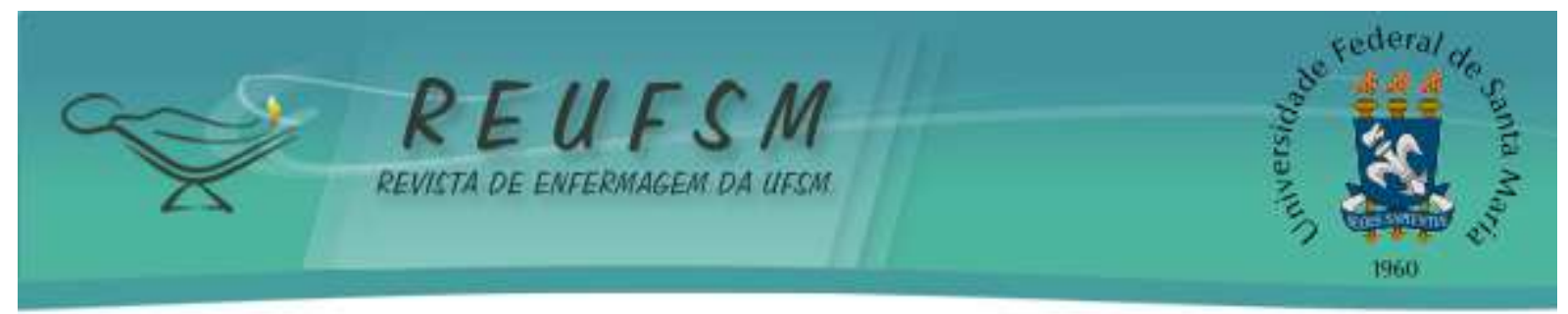

9. Bardin L. Análise de conteúdo. $5^{\text {a }}$ ed. Lisboa: Edições 70; 2011. 229p.

10. Lima DWC, et al. Referenciais teóricos que norteiam a prática de enfermagem em saúde mental. Esc Anna Nery Rev Enferm 2014 jun [acesso em 2017 abr 02];18(2):33642. Disponível em: http://www.scielo.br/pdf/ean/v18n2/1414-8145-ean-18-02-0336.pdf.

11. Amorim AMMNE, Cruz DKRC, Cardoso MLLO. Percepção do enfermeiro no cuidar ao doente mental: uma revisão de literatura. Rev Multip Saúde HSM [Internet]. 2013 abr [acesso em 2017 mar 24];1(2):53-62. Disponível em: http://ojs.saomarcos.org.br/ojs/index.php/cientifica/article/view/2/20.

12. Seabra P, Sá L. Fatores determinantes para as necessidades em saúde das pessoas consumidoras de drogas: uma revisão bibliográfica. Rev Port Enferm Saúde Mental [Internet]. 2011 jun [acesso em 2017 abr 02];(5):22-9. Disponível em: http://www.scielo.mec.pt/scielo.php?script=sci_arttext\&pid=S1647-

$21602011000100004 \& \operatorname{lng}=$ pt doi:10400.14/14456.

13. Silva MLB, Dimenstein MDB. Manejo da crise: encaminhamento e internação psiquiátrica em questão. Arq Bras Psicol [Internet]. 2014 [acesso em 2017 abr 17];66(3):3156. Disponível em: http://pepsic.bvsalud.org/pdf/arbp/v66n3/04.pdf.

14. Moghadam FM, Khoshknab MF, Pazargadi M. Psychiatric Nurses' Perceptions about Physical Restraint; A Qualitative Study. IJCBNM [Internet]. 2014 [acesso em 2018 jan 9];2(1):20-30. Disponivel em: https://www-ncbi-nlm-nihgov.ez76.periodicos.capes.gov.br/pmc/articles/PMC4201183/pdf/ijcbnm-2-20.pdf.

15. Wynn R. The Use of Physical Restraint in Norwegian Adult Psychiatric Hospitals. Psychiatry J [Internet]. 2015 [acesso em 2018 jan 9];2015(347246). Disponivel em: https://www-ncbi-nlm-nihgov.ez76.periodicos.capes.gov.br/pmc/articles/PMC4670873/pdf/PSYCHIATRY2015-347246.pdf.

16. Kondo EH, Vilella JC, Borba LO, Paes MR, Maftum MA. Abordagem da equipe de enfermagem ao usuário na emergência em saúde mental em um pronto atendimento. Rev Esc Enferm USP [Internet]. 2011 abr [acesso em 2017 abr 23];45(2):501-7. Disponível em: http://www.scielo.br/scielo.php?script=sci_arttext\&pid=S0080-62342011000200028.

17. Lima DWC, Vieira AN, Silveira LC. A escuta terapêutica no cuidado clínico de enfermagem em saúde mental. Texto \& Contexto Enferm [Internet]. 2015 mar [acesso em 2017 abr 23];24(1):154-60. Disponível em: http://www.scielo.br/pdf/tce/v24n1/pt_01040707-tce-24-01-00154.pdf.

18. Lima DWC, Silveira LC, Vieira AN. Listening in the treatment of psychological stress an integrative review. Rev Enferm UFPE [Internet]. 2012 set [acesso em 2017 abr 23];6(9):227380.

Disponível

em: http://www.revista.ufpe.br/revistaenfermagem/index.php/revista/article/view/2632/pdf_1479.

19. Bard ND, Antunes B, Roos CM, Olschowsky A, Pinho LB. Estigma e preconceito: vivência dos usuários de crack. Rev Latinoam Enferm [Internet]. 2016 [acesso em 2017 jun 03];24:e2680. Disponível em: http://www.scielo.br/pdf/rlae/v24/pt_0104-1169-rlae-0852-2680.pdf.

20. Paes MR, Silva TL, Chaves MMN, Maftum MA. O papel do hospital geral na rede de atenção à saúde mental no Brasil. Ciênc Cuid Saúde [Internet]. 2013 abr [acesso em 2017 jun 26];12(2):407-12. Disponível: http://dx.doi.org/10.4025/cienccuidsaude.v12i2.14207. 


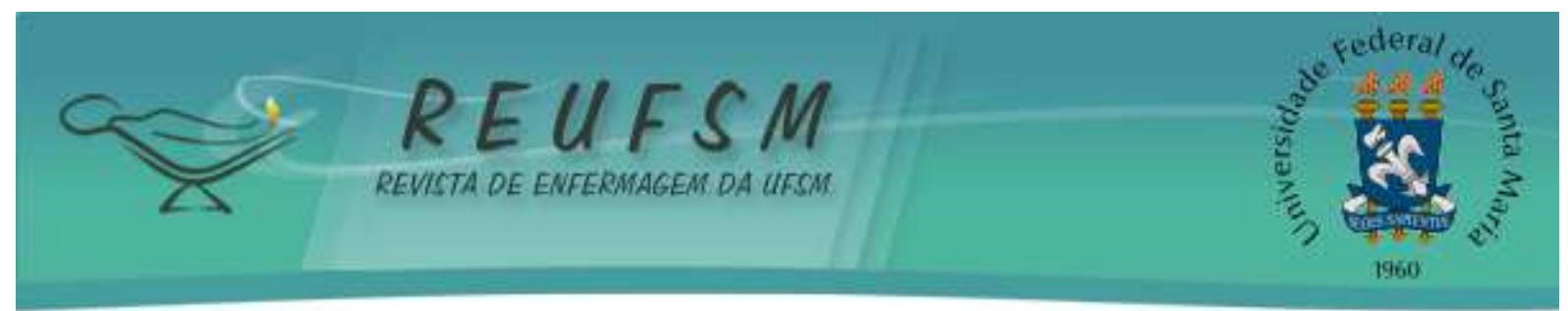

21. Ferreira GMK, Marins MF, Moraes RS, Lopes CLAS. Internações psiquiátricas em hospital geral e a política de saúde mental. J Nurs Health [Internet]. 2012 [acesso em 2017 jul 02];2(Suppl):333-9.

Disponível: https://periodicos.ufpel.edu.br/ojs2/index.php/enfermagem/article/view/3477/2862.

22. Brasil. Ministério da Saúde. Portaria $n^{\circ} 148$, de 31 de janeiro de 2012. Define as normas de funcionamento e habilitação do serviço hospitalar de referência para atenção a pessoas com sofrimento ou transtorno mental e com necessidades de saúde decorrentes do uso de álcool, crack e outras drogas, do componente hospitalar da rede de atenção psicossocial, e institui incentivos financeiros de investimento e de custeio. Brasília (DF): Ministério da Saúde; 2012. [acesso em 2017 jun 07]. Disponível em: http://bvsms.saude.gov.br/bvs/saudelegis/gm/2012/prt0148_31_01_2012.html.

23. Fiorati R, Saeki T. As dificuldades na construção do modo de atenção psicossocial em serviços extra-hospitalares de saúde mental. Saúde Debate [Internet]. 2013 jun [acesso em 2017 jun 07];37(97):305-12. Disponível em: http://www.scielo.br/pdf/sdeb/v37n97/v37n97a12 .pdf.

24. Mello AL, Backes DS, Terra MG, Rangel Rf, Nietsche EA, Salbego C. (Re) pensando la educación continua en las nuevas metodologias de intervención en salud. Rev Cubana Enferm [Internet]. 2017 [acesso em 2018 jan 9];33(3). Disponivel em: http://revenfermeria.sld.cu/index.php/enf/article/view/1104/285.

25. Guimarães AN, Borba LO, Maftum MA, Larocca LM, Nimtz MA. Mudanças na atenção à saúde mental decorrentes da reforma psiquiátrica: percepções dos profissionais de Enfermagem. Ciênc Cuid Saúde [Internet]. 2015 jan [acesso em 2017 jul 02];14(1):830-8. Disponível: http://dx.doi.org/10.4025/cienccuidsaude.v14i1.22187.

Data de submissão: 18/10/2017

Data de aceite: 06/02/2018

Autor correspondente: Deivson Wendell da Costa Lima

Email: deivsonwendell@hotmail.com

Endereço: Rua Desembargador Dionísio Filgueira, 383, Centro, Mossoró - RN.

CEP: $59610-090$. 